\title{
Using statistical learning to close two-fluid multiphase flow equations for bubbly flows in vertical channels
}

\author{
Ming Ma, Jiacai Lu, Gretar Tryggvason* \\ ${ }^{a}$ Department of Aerospace and Mechanical Engineering, University of Notre Dame, Notre \\ Dame, IN 46556, USA
}

\begin{abstract}
Data generated by direct numerical simulations (DNS) of bubbly up-flow in a periodic vertical channel is used to generate closure relationships for a simplified two-fluid model for the average flow. Nearly spherical bubbles, initially placed in a fully developed parabolic flow, are driven relatively quickly to the walls, where they increase drag and slowly reduce the flow rate. Once the flow rate has been decreased enough, some of the bubbles move back into the channel interior and the void fraction there approaches the value needed to balance the weight of the mixture and the imposed pressure gradient. A database is generated by averaging the DNS results over planes parallel to the walls, and a Model Averaging Neural Network (MANN) is used to find the relationships between unknown closure terms in a simple model equations for the average flow and the resolved variables. The closure relations are then tested, by following the evolution of different initial conditions, and it is found that the model predictions are in reasonably good agreement with DNS results.
\end{abstract}

Keywords: Bubble dynamics, Gas/liquid flow, DNS, Two fluid models, Closure terms, Model averaging neural networks, Statistical learning

\footnotetext{
* Corresponding author

Email address: gtryggva@nd.edu (Gretar Tryggvason)
}

Preprint submitted to International Journal of Multiphaseflow

December 23, 2015 


\section{Introduction}

For many multiphase flows the physics is well understood in the sense that the dynamics is believed to be accurately described by the Navier-Stokes equations. The large range of temporal and spatial scales in industrial size systems does, however, make solutions of the equations impractical, even on modern supercomputers. For many purposes a low order description of the system, such as the average motion, is all that is needed. A reduced order description can be derived by averaging or filtering the Navier-Stokes equations, but the process introduces unknown closure terms that account for the effect of the unresolved scales on the resolved ones. Those have to be modelled. The fundamental assumption behind modelling of multiphase flows is that the closure terms appearing in the equations for the averaged or filtered quantities depend on the averaged flow, such as the deformation tensor and the void fraction, and sometimes on some integral measures of the unresolved motion, such as the turbulent kinetic energy, dissipation rate, and surface area density. The latter quantities evolve by their own equations with their own closure terms that need to be related to other quantities, in the same way as for the equations for the average flow. The averaged and filtered equations can be written in several different forms and depending on the exact form we need different closure relations. For reviews of modelling of two phase flows see, for example, [1, 2, 3, 4] and [5].

Here we examine an infinitely long vertical channel with flow driven by an imposed pressure gradient and gravity. The average flow is homogeneous in the streamwise and spanwise direction and the averaged quantities depend only on the wall-normal coordinate, as well as time. The averaged equations were exam25 ined analytically for laminar bubbly flow by [6] and later by [7]. For turbulent flow 8] solved the equations for the average flow using asymptotic analysis, and numerical solutions have been reported by a number of investigators, including [9, 10, 11, 12. The results, both for laminar and turbulent flows, generally agree with the flow structure seen experimentally, once the model coefficients have 
velocity and void fraction in the core, wall peaking for upflow and a wall-layer without bubbles for downflow.

In 13 we used neural networks to obtain closure relationships for laminar bubbly upflows in fully periodic domains from DNS results and found that the relationships allowed us to predict the evolution of different initial conditions (velocity and void fraction profiles) using a simple two-fluid model for the average flow. Here we examine the evolution of laminar bubbly flows in vertical channels, again using DNS results to find closure relationships for the average two-fluid model by model averaging neural networks. Although the flow is similar to the one examined in our earlier paper, the presence of walls adds considerable complexity. First of all, and even though the governing parameters are chosen so that the bubbles remain nearly spherical, surface tension effects are important near the wall and must be included in the average model, resulting in new terms that must be closed. Secondly, finding the neural network coeffi45 cients for the relationships between the closure terms and the resolved average variables is much more difficult and we have had to adopt new strategies to train the model. Since the bubbles are driven to the wall relatively quickly at the early stage, but the flow then changes relatively slowly, new data sampling strategies have been used. Those strategies are discussed in the method section.

Applications of various statistical techniques have a long history in studies of complex flows ([14, for example). As the use of such techniques has become more widespread for a large range of other problems, ranging from predicting consumer preferences to identifying security threats, methods for statistical learning have advanced rapidly. For turbulence flows, applications include the development of an adaptive controller for drag reduction by [15, and various reconstructions of the flow. [16] used neural networks to reconstruct near-wall turbulent flows and [17] used neural network to interpolate DNS data for a backward facing step. Other applications include the use of statistical learning to quantify the uncertainty in model predictions. [18 used Bayesian probabilistic ${ }_{60}$ approach to quantify the uncertainty in RANS model predictions for turbulent channel flow, [19] examined the uncertainty for turbulent boundary layers, and 
[20] used machine learning to compare RANS results with DNS and LES predictions to identify regions of high uncertainty, presumably where the assumptions behind the RANS model no longer hold. For our purpose, the use of statistical learning to automate, at least partially, the development and evaluation of reduced order models, is most relevant. For turbulent flows, examples includes the development of a subgrid model for LES by [21, the optimal estimation of subgrid models for LES by [22, the parametrization of surface features in coarse LES by [23, and the use of neural networks to optimize the model constants of the $k-\varepsilon$ turbulence model applied to simulations of data centres by 24]. Most recently, 25] have used statistical learning to determine the functional dependency of the closure terms for data generated by Spalart-Allmaras turbulence model, rather than full DNS, and [26] and 27] have used inverse modelling to obtain spatially distributed functional terms to aid closure modelling, instead of inferring model parameters directly. For multiphase flows, 28, computed the response of fully resolved particles to shocks and used a neural network to develop closure laws for macroscopic simulations of the gas-particle mixture. 29] discuss various techniques to bridge the scales between detailed microscopic simulations and macroscopic models, focusing on the convergence rate for various model problems.

\section{Problem Setup}

We examine bubbly flows in a rectangular vertical channel, between two vertical plates, with periodic boundaries in the streamwise and spanwise direction, as sketched in figure 1. At steady state the flow of nearly spherical bubbles has a simple structure, consisting of a nearly homogeneous core region and a thin bubble-rich wall-layer ([30]). The structure is determined by the lift forces on the bubbles, which drive them toward the side where the relative liquid velocity is largest, in a frame of reference moving with the bubble. Thus, in upflow the bubbles are driven toward the walls. Studies of the magnitude of the lift force and how it depends on the shear and other properties of the flow include [31, 32 . 


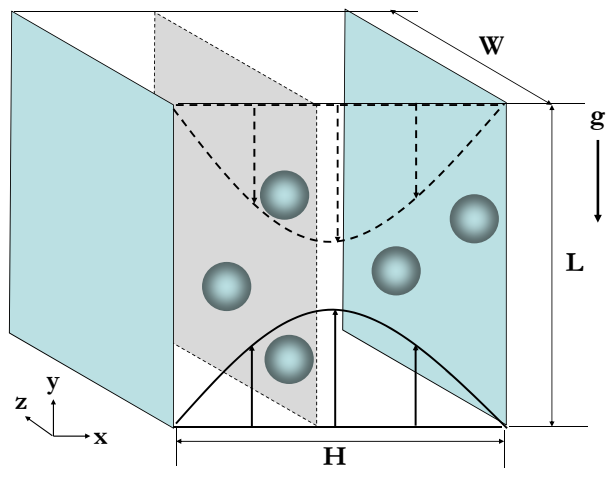

Figure 1: A sketch of the computational domain. The domain is bounded by vertical walls and is periodic in the other directions.

and 33. The lift force depends strongly on the deformability of the bubble and larger bubbles (and thus more deformable) tend to experience either little lift or a lift in the opposite direction from the nearly spherical ones ([34; 35]). Very little is known about the lift on many bubbles in high void fraction flows, although preliminary studies can be found in [36]. The horizontal migration of bubbles from the core to the wall increases the average density of the mixture in the core and if there are enough bubbles the weight of the mixture eventually balances the imposed pressure gradient and as the shear becomes zero the migration of bubbles stops. The void fraction in the core and the wall layer are therefore easily predicted. One of the main conclusions of these studies is that the steady state flow does not depend on the magnitude of the lift coefficientonly its sign. This suggests that if we intend to use the DNS results to evaluate and help construct models of the average flow, it is necessary to examine the transient motion. Initial efforts to do so can be found in [37, who studied the transient motion of bubbles in a vertical Couette flow.

The problem specification is similar to 13 . The width of the domain, in the wall normal direction is $H=2$, in the stream wise direction the length is $L=1.5$ and the span wise width is $W=1.0$. To make the computations as easy as possible, the density and viscosity are taken to be $\rho_{l} / \rho_{g}=2.5 / 0.125$; 
and $\mu_{l} / \mu_{g}=0.01 / 0.005$, where the subscript $l$ denotes the liquid and $g$ stands for the gas. Gravity acceleration is $g_{y}=1.0$ and total pressure gradient in the streamwise direction is $\beta=-0.04$, where $\beta=d p_{o} / d y+\rho_{a v g} g_{y}$. The flow contains $N=49$ bubbles of diameter $d_{e}=0.18$, giving an average void fraction of $\alpha_{\text {avg }}=0.049$. The surface tension coefficient is $\sigma=0.4$. These parameters result in a Morton number of $M=g_{y} \mu_{l}^{4} / \rho_{l} \sigma^{3}=2.56 \times 10^{-8}$ and an Eötvös number of $E o=\rho_{l} g_{y} d^{2} / \sigma=0.2025$. The initial velocity is a parabolic profile

$$
U(y)=\frac{H^{2} \beta}{2 \mu_{l}}\left[\left(\frac{y}{H}\right)^{2}-\frac{y}{H}\right],
$$

where the wall shear balances the pressure gradient and the weight of the mixture. The maximum velocity is $U_{\max }=3.00$, and the bulk velocity of the channel is $U_{b}=2.0$. The channel Reynolds number based on the bulk velocity and width of the channel is 1000 . The domain is resolved by $192 \times 192 \times 128$ grid points in the stream wise, wall-normal and span wise direction. The grid is stretched in the wall-normal direction to increase the resolution at the wall. For further details, see [30]. At steady state, the average wall shear stress $\tau_{w}$ is related to the pressure gradient and the weight of the bubble/liquid mixture by a streamwise momentum balance:

$$
\tau_{w}=-\left(d P_{0} / d z+\rho_{a v g} g_{y}\right) h=-\beta H / 2,
$$

which is found by taking a simple force balance for the whole domain. Since the liquid and the bubbles are incompressible, $\rho_{\text {avg }}$ is constant, and $\beta$ is therefore constant, since we assume that the imposed pressure gradient does not change. The direction of the flow depends directly on the sign of $\beta$ and for upflow we must have $\beta<0$.

The Navier-Stokes equations are solved by an explicit projection method that is second order in time, using a fixed, staggered grid where the advection terms are resolved by a QUICK scheme and the viscous terms by a second order centred approximation. In order to keep the boundary between the different fluids sharp, to advect the density and the viscosity fields, and to accurately compute the surface tension, the fluid interface is tracked by connected marker 
points (the "front"). The front points, which are connected to form an unstructured surface grid, are advected by the fluid velocity, interpolated from the fixed grid. As the front deforms, surface markers are dynamically added

and the average vertical velocity as functions of the wall-normal coordinate, we integrate the mass and momentum equations over planes parallel to the walls, 
taking the density and the viscosity of the gas to be zero. The averaging is essentially the same as is worked out in detail in [13, except here we do not assume that the bubbles remain completely spherical. We define the average liquid volume fraction and the average phasic vertical liquid velocity by

$$
\alpha_{l}=\frac{1}{A_{z y}} \int \chi d a, \quad\left\langle v>_{l}=\frac{1}{\alpha_{l} A_{z y}} \int \chi v d a .\right.
$$

where $\chi$ is an indicator function such that $\chi=1$ in the liquid phase and $\chi=0$ in the gas. $A_{z y}$ is the area of a plane parallel to the walls, as sketched in figure 1. The results of the averaging are two equations for the liquid volume fraction and the average vertical velocity of the liquid. The equation for the average liquid volume fraction is:

$$
\frac{\partial \alpha_{l}}{\partial t}+\frac{\partial F_{l}}{\partial x}=0
$$

The horizontal flux of liquid is given by

$$
F_{l}=\frac{1}{\alpha_{l} A_{z y}} \int \chi u d a=\alpha_{l}<u>_{l} .
$$

The gas volume fraction, $\alpha_{g}$, and average horizontal velocity, $\langle u\rangle_{g}$, are found in the same way. Adding the equations for the gas and the liquid, using that the volume fractions must add up to unity, $\alpha_{l}+\alpha_{g}=1$, and that the $u$ velocities at the walls are zero, shows that

$$
F_{g}+F_{l}=\alpha_{g}<u>_{g}+\alpha_{l}<u>_{l}=0 \text {. }
$$

Thus, gas flow in one horizontal direction is always balanced by liquid flow in the opposite direction.

The averaged equation for the vertical liquid velocity $\langle v\rangle_{l}$ is:

$$
\begin{gathered}
\frac{\partial}{\partial t} \alpha_{l}<v>_{l}+\frac{\partial}{\partial x} \alpha_{l}<u>_{l}<v>_{l}= \\
-\frac{1}{\rho_{l}} \frac{d p}{d y}-\frac{1}{\rho_{l}} \alpha_{l} \rho_{l} g_{y}+\frac{1}{\rho_{l}} \frac{\partial}{\partial x}\left(\alpha_{l} \mu_{l} \frac{\partial<v>_{l}}{\partial x}\right)-\frac{\partial}{\partial x}\left(\alpha_{l}<u^{\prime} v^{\prime}>_{l}\right)+\left(f_{\sigma}\right)_{y} .
\end{gathered}
$$

where the last term is the average surface tension. Notice that the momentum equation contains the liquid fluxes since the average horizontal liquid velocity is given by $\alpha_{l}<u_{l}>=F_{l}$. Equations (4) and (7) give the evolution of $\alpha_{l}$ 
and $\langle v\rangle_{l}$, but require closure relations for $F_{l},<u^{\prime} v^{\prime}>_{l}$ and $\left(f_{\sigma}\right)_{y}$. The averaged equations are solved by a simple first order upwind method using a large number of grid points and very small time steps to ensure that the results have converged. The code has been written in $\mathrm{R}$, to simplify the incorporation of the functional relationships for the closure terms (see below).

The data generated by the DNS is averaged over planes parallel to the walls, resulting in a database containing the average gas flux, liquid streaming stresses, and surface tension, along with void fraction, void fraction gradient, gradient of the average vertical liquid velocity, and the distance to the nearest wall, at the horizontal grid point, at all time intervals. We now assume that each of the first three quantities depends uniquely on the latter four, at least in some average sense (since we expect considerably scatter):

$$
F_{g}=f(\mathbf{x}), \quad<u^{\prime} v^{\prime}>_{l}=g(\mathbf{x}), \quad\left(f_{\sigma}\right)_{y}=s(\mathbf{x})
$$

where

$$
\mathbf{x}=\left(\frac{\partial<v_{l}>}{\partial x}, \alpha_{g}, \frac{\partial \alpha_{g}}{\partial x}, d_{w}\right)
$$

The functions $f, g$, and $s$ are determined using the DNS database. While these functional relationships can be found in many ways, here we use Model Averaging Neural Networks (MANN). The learning strategy for the Neural Networks part is similar to [13], since we expect the relationships to be nonlinear. The unknown coefficients are initially set to small values near zero and and then adjusted by an iterative process. Since the iterations are stopped before they have reached a final converged value, to avoid over-fitting, different initial values can lead to different fits. To reduce the variance between training sessions with different initial values two techniques are combined here: in the first method we create several NN models using different randomly selected starting coefficients, and aggregate all the NN models to generate the averaged NN fit. This procedure has been shown to result in more stable predictions $([39,40,41])$. The other technique is to use resampling and bagging the data during the training of each NN, where we resample the training data with replacement for each NN's training dataset before aggregating all the models, and this has been shown 
to result in improvements for unstable procedures like $\mathrm{NN}$ and decision tree method $([42,43])$. Both MANN and data resampling techniques are available in the Caret package, implemented in $\mathrm{R}(44])$.

During the training, we pool all the spatial and temporal data together for the upflow channel case, and, unlike for the fully periodic case ([13]) where all the data is sampled uniformly at the early time, here we select the frequency of the training data following a exponential distribution based on time, since for the upflow case considered here, the bubbles are initially driven relatively quickly to the wall, but at the late stage the flow evolves very slowly. The final $\mathrm{NN}$ is averaged by $5 \mathrm{NNs}$, each with a 3 layer structure: 4 inputs, 12 neurons and 1 output for each unknown closures. All the unknown coefficients for each NN start randomly below 0.1 and the maximum number of iteration for each NN's training is 250 . We have tested the "tuning" parameters such as the range of small starting random numbers, number of neurons, iteration steps, and the number of NNs needed before aggregation, and selected the numbers given here for the best performance.

Neural networks are, of course, not the only statistical learning technique that can be used, and we have also experimented with linear regressions. Unlike MANN, where the functional form of the closure terms are unknown, here we 235 postulate a functional form for the relationship with unknown coefficients and determine the unknown coefficients from the DNS data. We assume that the formula for gas flux is:

$$
F_{g}=C\left(-\operatorname{sign}\left(\frac{d<v>_{l}}{d x}\right)\right) \alpha_{g}^{m}\left(\frac{d<v>_{l}}{d x}\right)^{n} d(x)^{p} .
$$

The direction of the gas flux is modelled by adding the $-\operatorname{sign}\left(d<v>_{l} / d x\right)$ term, since the sign the of the gas flux is determined by the direction of the lift force. Considering the gas flux to be correlated with $\alpha_{g}, d<v>_{l} / d x$ and $d(x)$, the distance to the nearest wall, we propose a nonlinear closure with unknown powers. We find the unknown parameters from the same DNS data used for neural networks' training and use the bootstrap method to reduce the uncertainty of the training results. We note that we have not included the 

necessarily zero.

\section{Results}

Figure 2 shows the bubbles and the velocity field in the mid-plane through the domain at six times. The first frame is at time zero where the flow is parabolic and the bubbles are spread nearly homogeneously across the channel. In the next frame, most of the bubbles have moved toward the wall. There is a layer of bubbles at both walls, and only a couple of bubbles in the middle. The bubble motion is fairly unsteady and the wall-layer is often broken up by the motion of the bubbles just outside of it. This development continues in the third frame, where the middle of the channel is mostly free of bubbles. In the fourth frame, all the bubbles are near the walls, and in frame five, the bubbles stay close to the wall, but the average liquid velocity is lower. In the sixth frame, at a much later time, some of the bubbles have moved back into the channel interior and the void fraction there approaches the value needed to balance the weight of the mixture and the imposed pressure gradient.

\subsection{Training of the model}

The results shown in figure 2 provide the training data for our model. Before we plug in the models for the closure terms into the averaged equations, we need a reliable training procedure for the neural networks. We collect various quantities averaged over planes parallel to the walls, together with the unknown closure terms, at different times and use neural networks for the fittings. Here we assume that the unknown closure terms in equations (6) and (7) depend only on the averaged quantities and not on any average description of the unresolved field. Obviously there cannot be any dependency on $\langle v\rangle_{l}$ directly, so to the lowest order we assume a dependency on the velocity gradient. The closure terms can, however, depend on the void fraction and we include the possibility that there is also a dependency on the gradient of $\alpha_{g}$. We have done a test of 

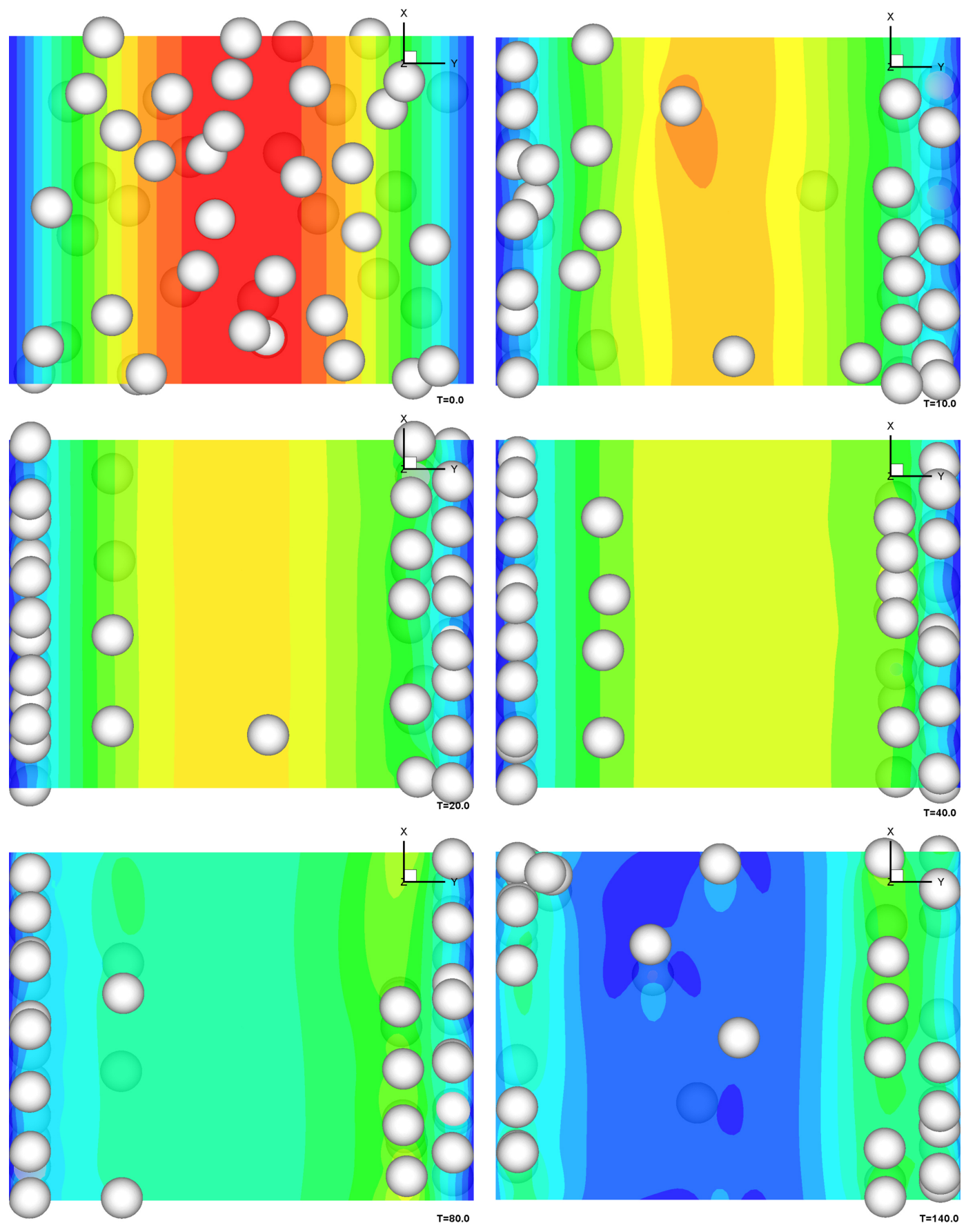

Figure 2: The bubble distribution at several different times, starting with the initial conditions in the top left corner. 
the model variable selection, and in $[13$ these three variables were found to be enough. For wall bounded flow we must also allow for a possible dependency on the distance to the wall $d_{w}$. Figure 3 shows the quality of the fitting. In the left column we show a histogram of the training error distribution for the gas fluxes, streaming stress and surface tension. Most of the training errors are clustered around zero, and the number of instance falls off rapidly as the error becomes larger. The distribution is nearly Gaussian, though there is a slight skewness. In the right column we show a scatter plot of the predicted average gas fluxes versus the average fluxes from the DNS for the test data. Although there is some spread, the points are mostly clustered around the forty five degree line. The output from the Caret package is a function with the relationship and we use these functions for each closure term in an $\mathrm{R}$ code to solve equations (6) and $(7)$. The predictions of the model are shown in figure 4 where we plot the average liquid velocity at five times in the left column and the void fraction at the same times in the right column, along with the averaged DNS results and from the model where the closure terms are found by linear regression. The results again show that when nearly spherical bubbles are injected into parabolic flow the evolution toward steady state is highly non-monotonic. First, all the bubbles migrate towards the walls, leaving the centre region nearly free of bubbles. Then the presence of the bubbles near the wall increases the friction there and reduces the flow rate. As the flow rate is reduced sufficiently some of the bubbles migrate back into the core region until the mixture there is in hydrostatic equilibrium. Thus, the initially parabolic velocity decreases rapidly in the middle of the channel and is nearly uniform across almost all of the channel at the last time shown, going to nearly zero only very near the walls. The void fraction profile is more complex but a careful inspection shows that the bubbles rapidly accumulate near the walls, with the void fraction going to zero in the middle of the channel and then returning to a profile consisting of nearly uniform value across most of the channel with peaks near the walls in a layer of thickness of about one bubble diameter. The initial migration of the bubbles to the wall takes place relatively fast, but the slowing down of the flow and the 

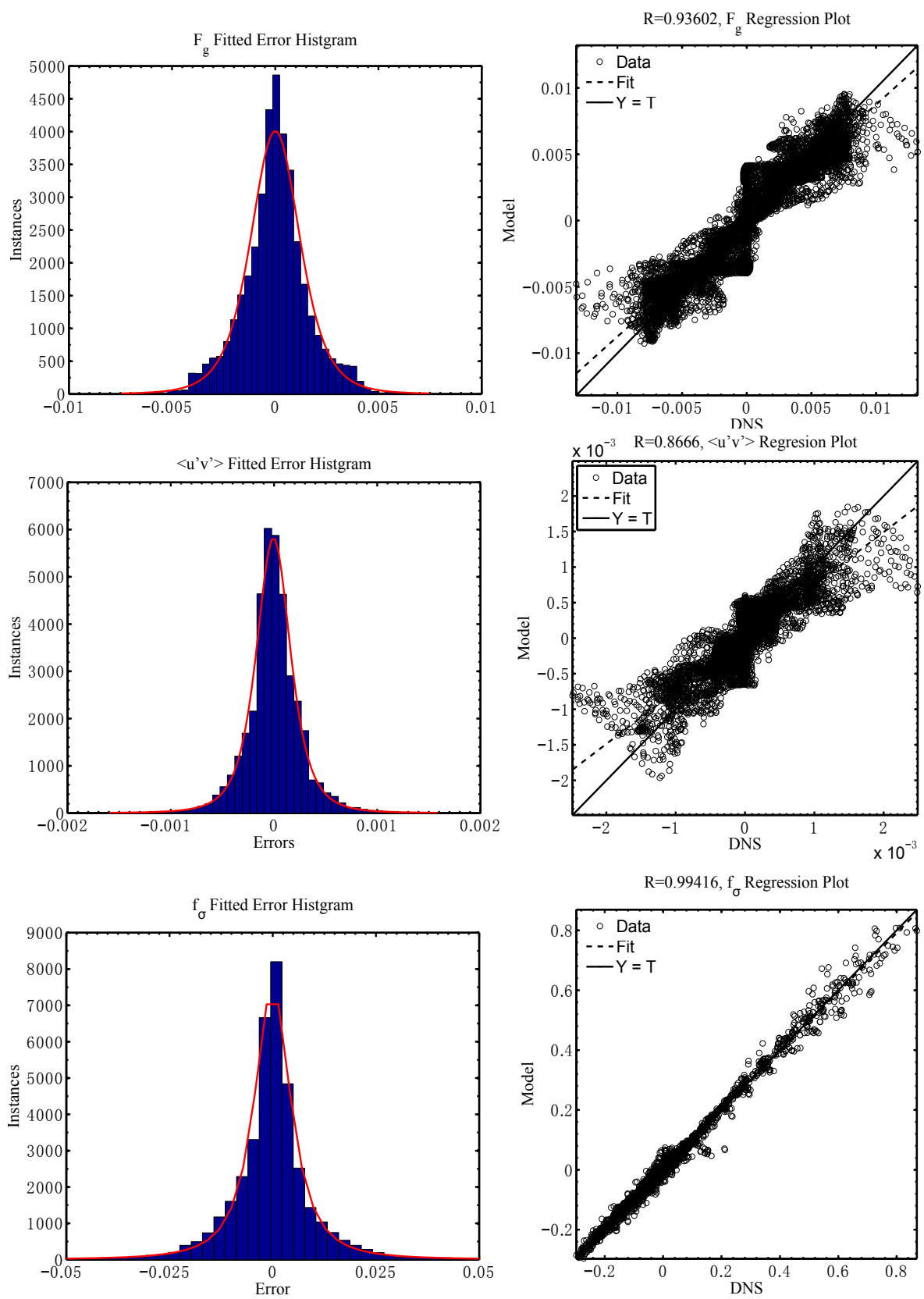

Figure 3: The training error histograms (left column) and the regression plots (right column) for the three closure terms: $F_{g},\left\langle u^{\prime} v^{\prime}\right\rangle$, and $f_{\sigma}$. 


\begin{tabular}{lcc} 
coefficients & Mean Value & Standard Deviation \\
\hline $\mathrm{C}$ & $2.84 \mathrm{e}-2$ & $2.24 \mathrm{e}-3$ \\
$\mathrm{~m}$ & $6.54 \mathrm{e}-1$ & $6.88 \mathrm{e}-2$ \\
$\mathrm{n}$ & $7.88 \mathrm{e}-1$ & $6.68 \mathrm{e}-2$ \\
$\mathrm{p}$ & 3.77 & $5.57 \mathrm{e}-1$
\end{tabular}

Table 1: The estimated regression coefficients and corresponding standard deviations, using bootstrap.

migration of the bubbles back into the core is a much slower process. Here we do not allow the bubbles to coalesce, but if they could then the migration to the wall might promote the formation of either larger bubbles that would move away from the wall relatively quickly or possibly a gas film at the wall. The linear regression is more sensitive to the data sampling techniques and data size than the MANN. Here a bootstrapping method is used for selecting the data with replacement for 2000 times (we gradually increase the data sample size from 500, and end at 2000), generating 2000 sub-dataset. In each subset we do a linear regression and then take the mean value of the unknown coefficients and estimate its standard deviation, in order to reduce the uncertainty and get more reliable results. Using bootstrap we can also estimate the standard deviation for each coefficient, as given in Table 1

In addition to directly checking the average void fraction and liquid velocity, we have also examined the unsteady evolution of the flow as shown in figure 5 . where we plot the average wall shear (top) and the average flow rate (bottom) as obtained from DNS data and from the model. Here the liquid flow rate $Q$ and average wall shear rate $\gamma$ are defined as:

$$
Q=\int_{0}^{H}<v>_{l} d x, \gamma=\left.\frac{d<v>_{l}}{d x}\right|_{x=0}
$$

Initially the shear stress is in balance with the steady state flow of the liquid but as bubbles accumulate near the walls, the wall shear increases and the flow starts to slow down. The wall shear quickly reaches maximum, when most of 

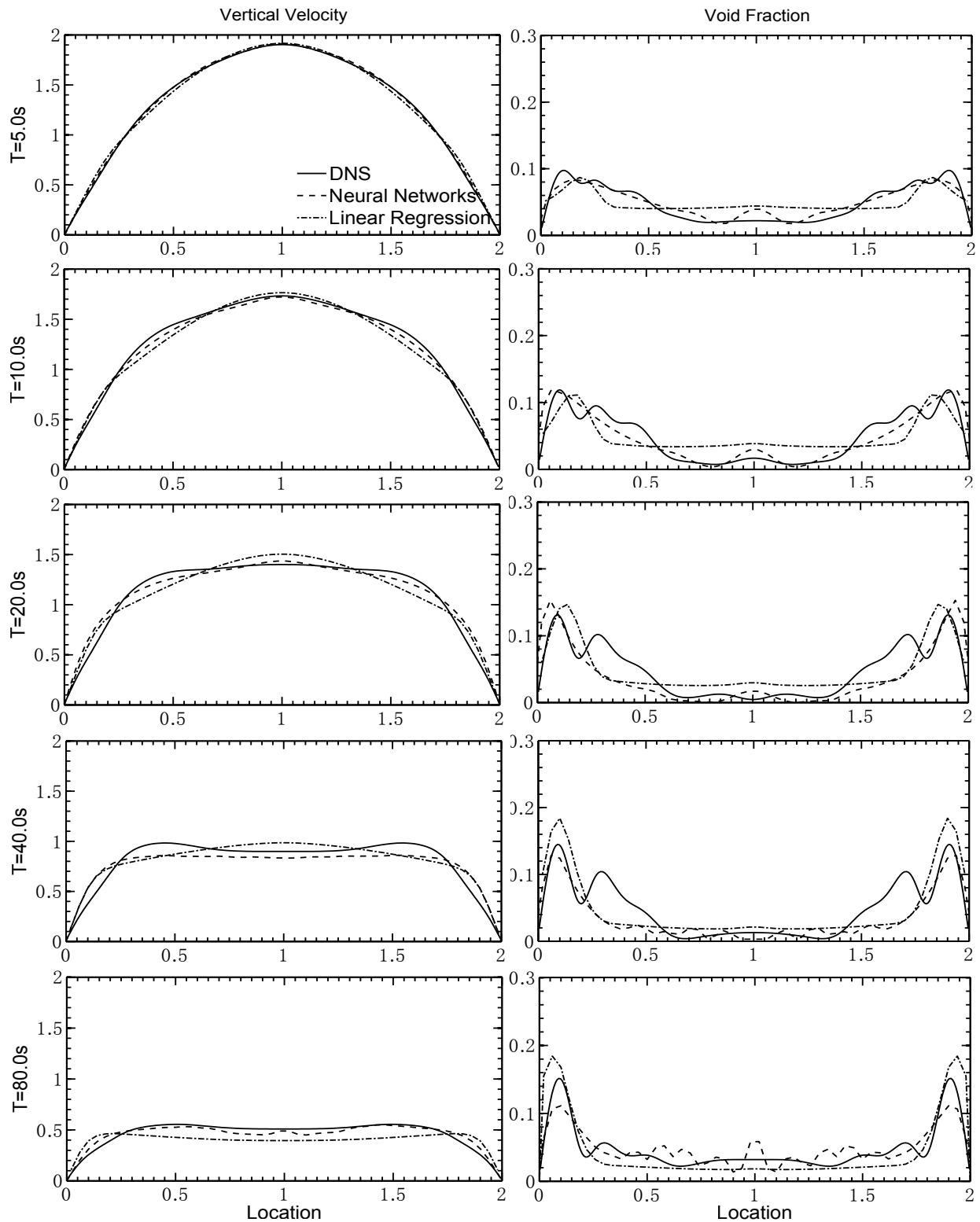

Figure 4: A comparison of the prediction of the model with averaged DNS data. The average liquid velocity is shown on the left, at several times, and the average void fraction on the right. 
Shear Rate at Wall

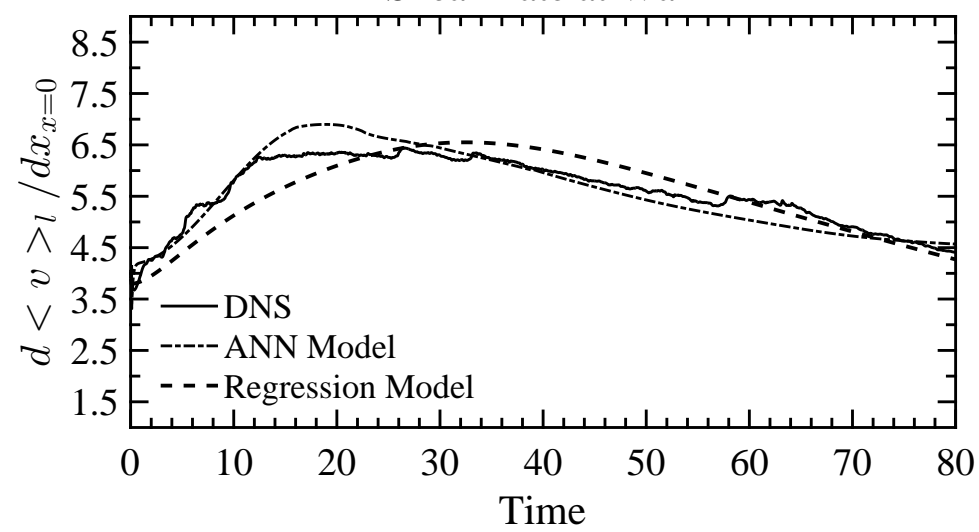

Liquid Flow Rate

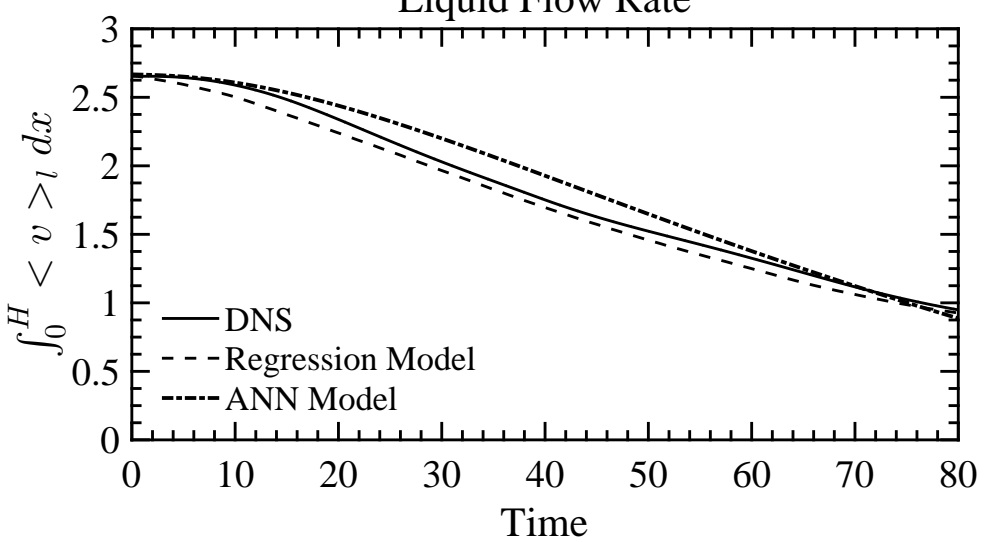

Figure 5: The average wall shear (top) and the flow rate (bottom) versus time, for the simulation shown in figure 4 
the bubbles are at the wall, and then drops slowly as the flow rate continues to

\subsection{Validation of the Model: applications to different initial conditions}

Ideally the relationship derived in the last section by MANN is sufficiently general so that it can be used for flows that are different from the "training" case. To examine how well it does, while still remaining within the rather restrictive for the upflow presented earlier. The average liquid velocity is shown in the left column and the void fraction at the same times in the right column, along with the averaged DNS results. The changes in the flow are far less dramatic than for the upflow. The bubbles move slightly closer to the channel centre and increase the buoyancy there, flattening the velocity profile, resulting in a nearly uniformly void fraction in the middle of channel and well-defined bubble free layers near the walls.

Similar to the upflow case, figure 7 shows a comparisons of the wall shear rate and the liquid flow rate, between DNS data and model predictions, for good and the minor differences observed are mainly during the early times. In general, if we train and test the model on the same case, we would expect a better prediction performance than testing the learned model on different cases. However, it is not always the case since we also need to consider the complexity

testing case, the closure results in predictions that are somewhat better than for the original training case.

The agreement between the model predictions and the averaged DNS date 

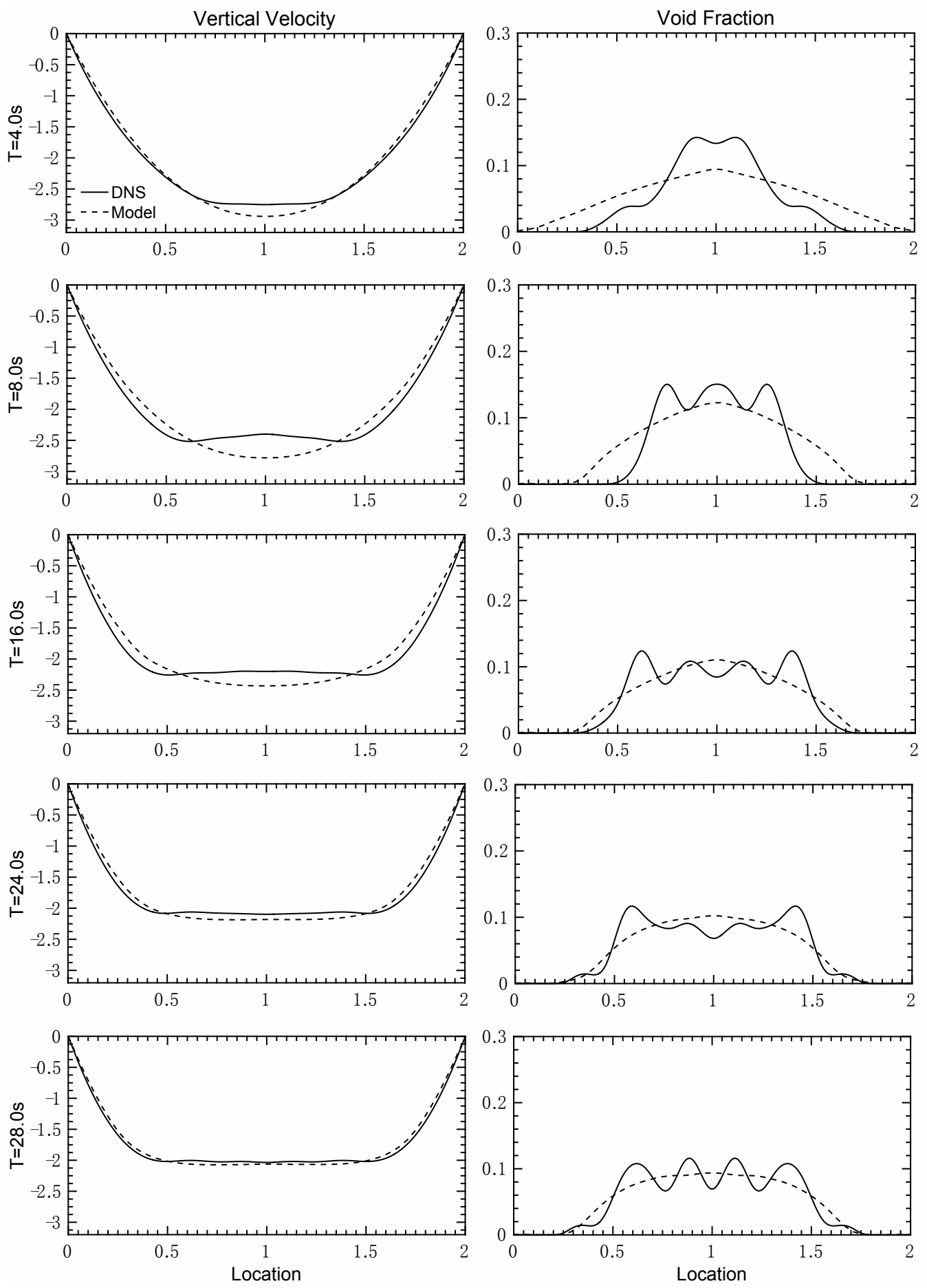

Figure 6: A comparison of model predictions 19 nd averaged DNS results for down flow. The average liquid velocity is shown on the left, at several times, and the average void fraction on the right. 

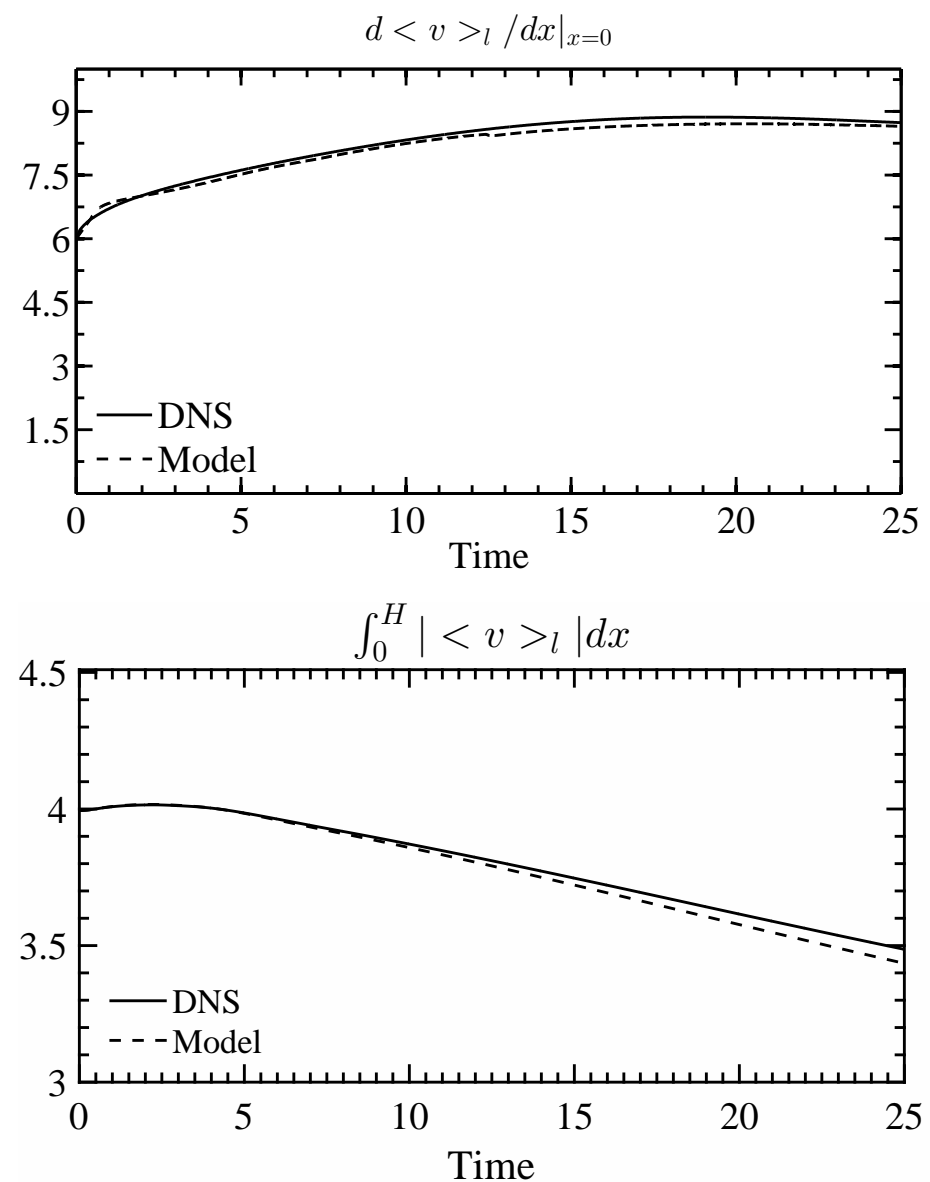

Figure 7: The average wall shear (top) and the flow rate (bottom) versus time for the simulation shown in figure 6 


\begin{tabular}{lcccc} 
Case & velocity & Initial void fraction & $M S D 1$ & $M S D 2$ \\
\hline Train & $2\left(-x^{2}+2 x\right)$ & uniform $\alpha_{g}=4.99 \%$ & 0.05661 & 0.002536 \\
Test 1 & $3\left(-x^{2}+2 x\right)$ & uniform $\alpha_{g}=4.99 \%$ & 0.08361 & 0.006262 \\
Test 2 & $2\left(x^{2}-2 x\right)$ & uniform $\alpha_{g}=4.99 \%$ & 0.01632 & 0.001637 \\
Test 3 & $3\left(x^{2}-2 x\right)$ & uniform $\alpha_{g}=4.99 \%$ & 0.03955 & 0.002374 \\
Test 4 & $\sin \pi x$ & uniform $\alpha_{g}=4.99 \%$ & 0.04801 & 0.006202 \\
Test 5 & $-x+1$ & uniform $\alpha_{g}=4.99 \%$ & 0.07569 & 0.004565
\end{tabular}

Table 2: The various initial conditions used to train and test the model for the closure terms. The last two columns are defined by equation 12 and 13 .

for other conditions, using the same closure relations, are similar. To summarize the results, we show in figure 8 the difference between the model and the DNS data versus time, for six cases, listed in Table 2. The results are condensed even further in the two rightmost columns in Table 2 where the mean square different of the error, defined by

$$
\begin{aligned}
M S D 1 & =\sqrt{\frac{1}{T} \int\left(\gamma_{D N S}-\gamma_{\text {model }}\right)^{2} d t}, \\
M S D 2 & =\sqrt{\frac{1}{T} \int\left(Q_{D N S}-Q_{\text {model }}\right)^{2} d t},
\end{aligned}
$$

In all cases the differences between the model predictions and the DNS data are of comparable order of magnitude, unlike the situation in [13, where the agreement is generally worse than for the training data. For wall bounded bubbly flow, the dynamics of the training case itself is more complex than for some of the testing cases, like the downflow ones. 

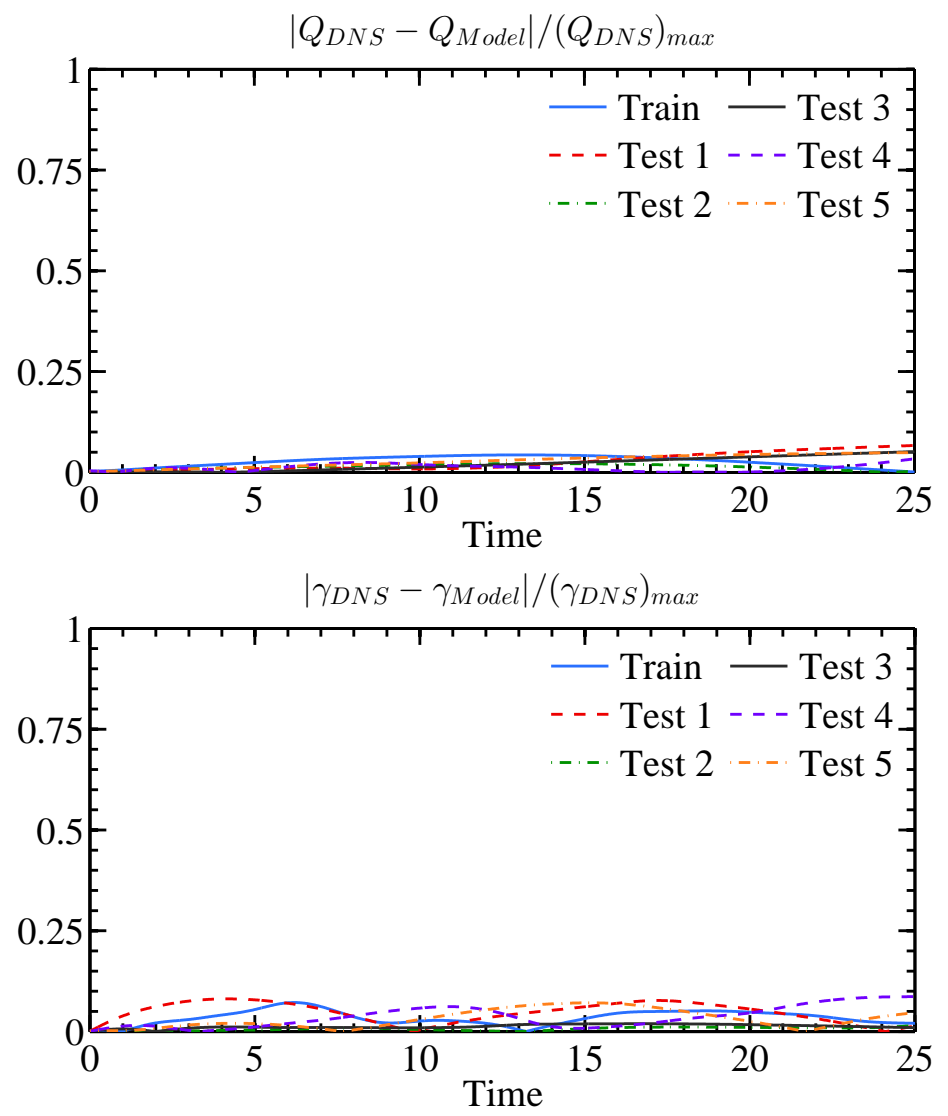

Figure 8: The difference between the liquid flow rate (top frame) and wall shear rate (bottom frame) predicted by the model and found from the DNS data versus time for all the initial conditions in Table 2 The differences have been normalized by the maximum value of liquid flow rate and wall shear rate in the DNS results. 


\subsection{Closure Terms Analysis}

365 numerical implementation of the model as functions that can be treated as a "black box." A visual inspection does, however, help us evaluate the quality of the fit and understand what physical processed are captured. In figure 9 and 10 we show how the gas fluxes, the streaming stresses and the average surface we set the void fraction equal to $\alpha_{g}=0.1$ and show the relationships as the distance to the wall gradually increases. In the bottom frame of figure 9 the distance to the wall is set to two bubble diameters and we show the flux for a few different void fractions. The wall distance function is linear and reaches with other types of wall distance function and not found much difference since the NN automatically adjusts the power of the wall distance function. For zero velocity gradient the gas fluxes are zero and for gradient near zero the gas fluxes are a decreasing function of the liquid shear rate, as we would expect for nearly spherical bubbles. The flux is lowest for bubbles close to the wall and the effects of the wall become smaller as $d_{w}$ increases. Eventually we would expect the wall to have no effect and the dashed line in figure 9 is a fit from simulations with no walls. Obviously the current results away from the walls agree well for low shear rates. As the shear rate is increased, however, the flux reaches a maximum and then decreases. This can, at least in principle, be due to two effects: The increased shear deforms the bubbles more and thus the lift changes sign, or this can be a result of the highest shear being found close to the walls, where the flux is reduced due to wall effects and not deformability. We suspect that it is the latter, as discussed below. 
Plot of $F_{g}, \alpha_{g}=0.1$

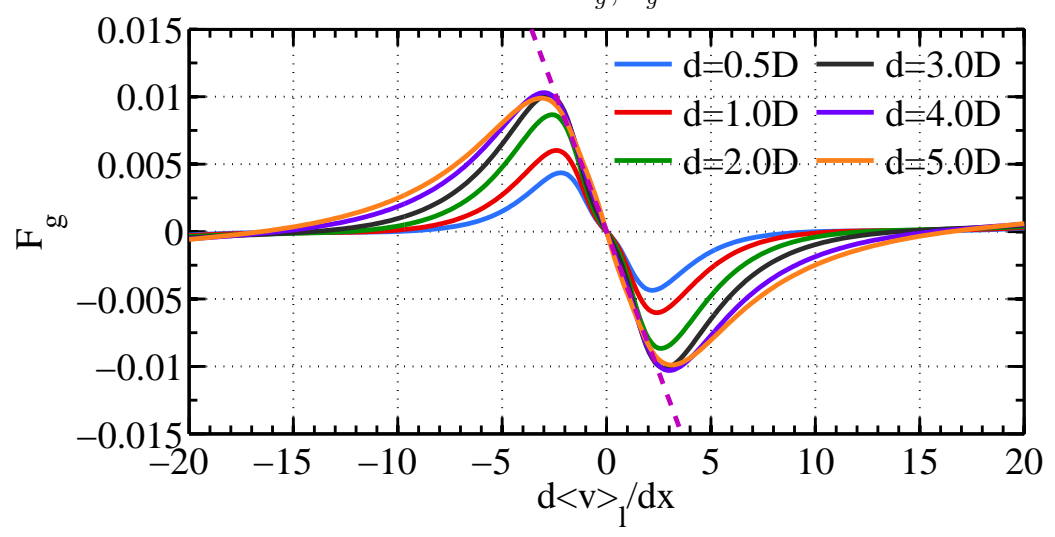

Plot of $F_{g}, d=2 D$

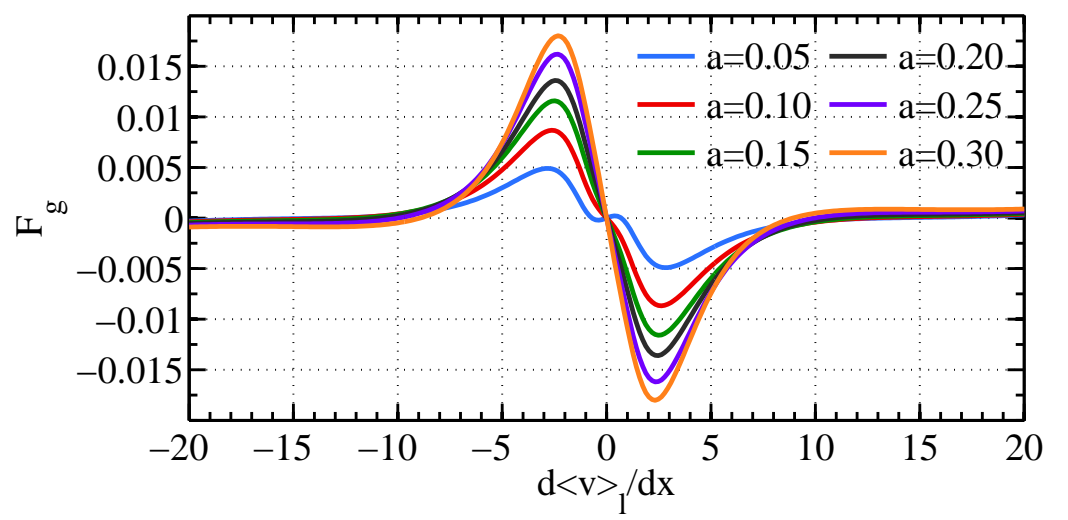

Figure 9: The closure term $F_{g}$ as a function of the liquid shear rate for different distances to the wall at a fixed value of void fraction $\alpha_{g}=0.1$ (top frame), and with different void fractions at a distance to the wall of two bubble diameters (bottom frame). 


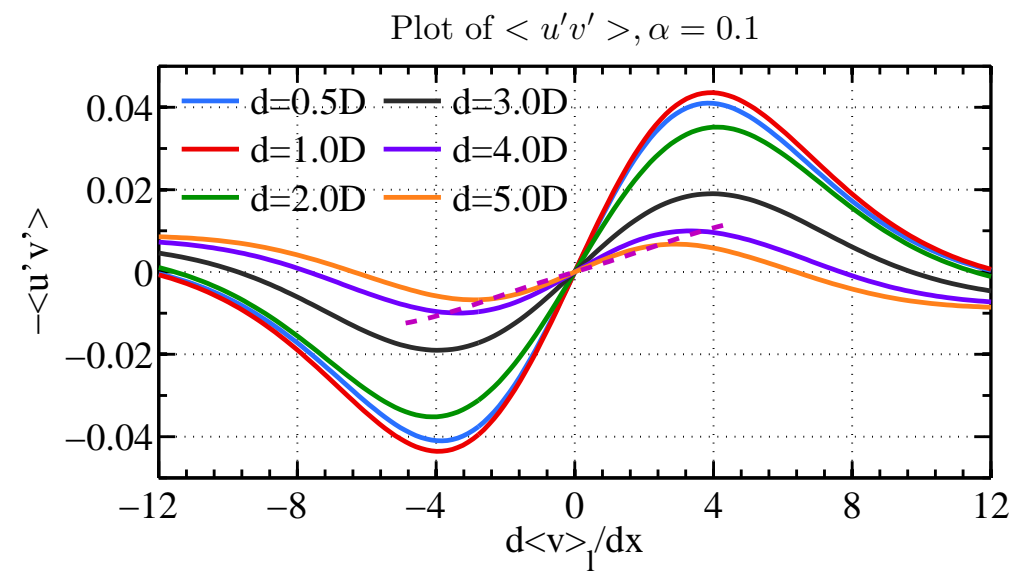

Plot of $f_{\sigma}, d=2 D$

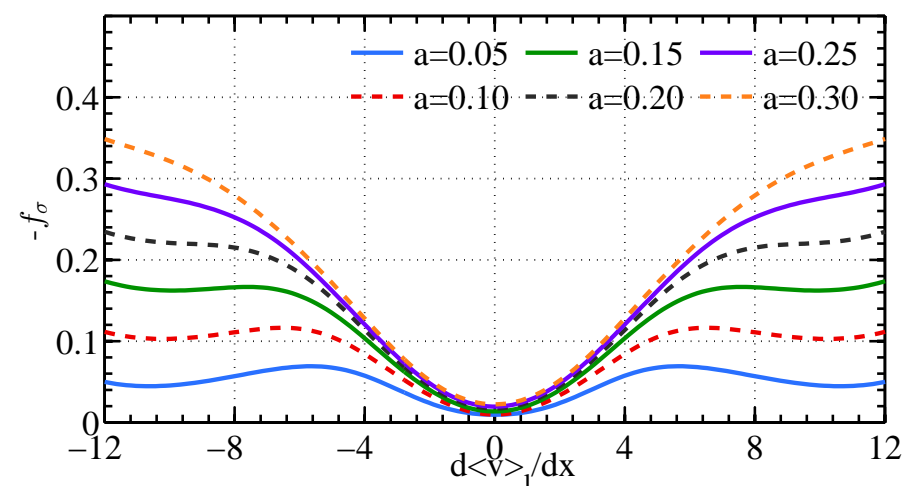

Figure 10: The closure term $\left\langle u^{\prime} v^{\prime}\right\rangle_{l}$ as functions of the liquid shear rate with different distances to the wall at a fixed value of void fraction $\alpha_{g}=0.1$ (top frame), and the closure term $f_{\sigma}$ with different void fractions at a fixed distance to the wall equal to two bubble diameters (bottom frame). 


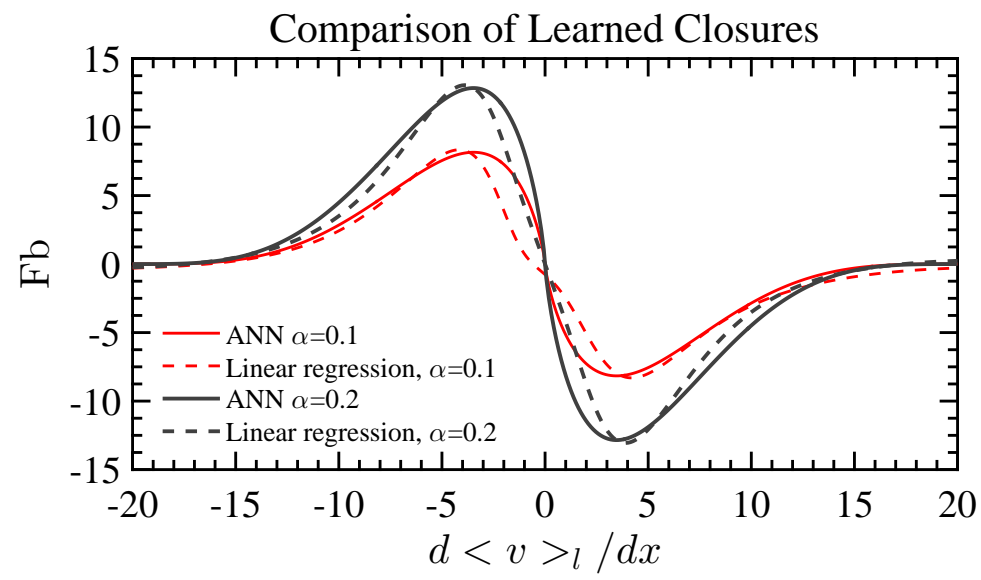

Figure 11: Comparisons of fitted closure term $F_{g}$ with MANN model (solid line) and linear regression model (dash line) at $\alpha_{g}=0.1$ (red line) and $\alpha_{g}=0.2$ (black line).

The fit for the streaming stress shows a similar trend. For zero shear the stress is zero and for low shear rate it is a decreasing functions of the liquid shear rate. It is lowest close to the wall and its depending on the distance to the wall is reduced as we move further away. As the top frame in figure 10 shows, the slop of the streaming stress is converging to a constant value in the low shear rate region, as the distance to the wall increases, and approaches the slope fitted by the periodic case without walls, shown by the dash line. The bottom frame of Figure 10 shows the average surface tension term versus the fluid shear for a distance to the wall equal to two bubble diameter. As the void fraction increases, more bubbles are near the wall, so the average surface tension increases. Looking at the fluxes directly can give us insights into the closure relationships and allow us to check if they are aligned with our intuition. Such an inspection can help us identify problems. In our case, for example, the gas fluxes go to zero for high shear rate for any distance to the wall. While it is true that high shear rates deform the bubbles and reduce lift and thus gas flux, the reason is much more likely to be that we do not have any data points for high shear away from the walls. We suspect that the similar behaviour for the streaming stress is for similar reasons. 
We have also examined the fluxed found using the linear regression and they are generally in good agreement with the fluxes found by the MANN, as shown in figure 11, where we plot the gas fluxes versus the shear rate in the liquid, for two values of the void fraction and a distance to the wall equal to two bubble diameters, as obtained by both approaches. Notice however that the linear regression neglect any dependency on the void fraction gradient, so we would not expect a perfect agreement.

\section{Conclusions}

Here we have examined how DNS results can be used to provide closure relations for a simple model of the average flow. We have done this in the context of a fairly simple setup, following the transient evolution of bubbly flow in a vertical channel. The bubbles are all of the same size, the initial liquid velocity is taken to be laminar and parabolic, and the Reynolds number is sufficiently low so that velocity disturbances are likely to be only due to the bubble motion. We use a neural network that is trained on one DNS data set and then applied to other situations where the initial velocity, void fraction and boundary conditions are different, but for which we also have DNS results to compare with. Overall, the average equations with the neural network closure reproduce the main aspects of the DNS results, including the generally rapid change initially, unsteadiness remaining after the initial adjustment and the final steadiness state.

We have also found the closure terms by postulating a relationship with unknown coefficients that are found using linear regression. Both approaches give comparable results, but given the slightly better accuracy and their generality, we believe that the neural networks are the preferred approach for more complex flows.

We emphasize that the system considered here, as well as the two-equation model, are relatively simple. Specifically, no average descriptions of unresolved quantities appear to be necessary (which might be governed by their own evolu- 
tion equations with unknown closure terms, like the turbulent kinetic energy).

Furthermore, the gas flux appears to be a function of the local state and no evolution equation for the bubble velocity needs to be solved. We have also examined a model where a new equation governing the evolution of gas flux term is added, and the averaged horizontal pressure gradient appears as a new closure terms instead of gas flux term. The three-equation model does not dramatically increase the prediction accuracy, at least not for the current parameter range. We close by noting that while most multiphase flows are considerably more complex than the system considered here, we believe that there is nothing that intrinsically limits using statistical learning for the closure terms to simple systems only.

\section{Acknowledgement}

This work is supported by the National Science Foundation Grant CBET1335913.

\section{References}

[1] M. Ishii, Thermo-Fluid Dynamic Theory of Two-Phase Flow, Eyrolles, 1975.

[2] I. Kataoka, A. Serizawa, Basic equations of turbulence in gas liquid twophase flow, Int. J. Multiphase Flow 12 (1989) 745-758.

[3] D. Z. Zhang, A. Prosperetti, Ensemble phase-averaged equations for bubbly flows, Phys. Fluids 6 (1994) 2956-2970.

[4] D. A. Drew, S. L. Passman, Theory of multicomponent fluids, Springer, 1999.

[5] A. Prosperetti, G. Tryggvason, Computational Methods for Multiphase Flow, Cambridge University Press, 2007. 
[6] S. P. Antal, R. T. Lahey, J. E. Flaherty, Analysis of phase distribution in fully developed laminar bubbly two-phase flows, Int. J. Multiphase Flow 15 (1991) 635-652.

[7] O. E. Azpitarte, G. C. Buscaglia, Analytical and numerical evaluation of two-fluid model solutions for laminar fully developed bubbly two-phase flows, Chem. Eng. Sci. 58 (2003) 3765-3776.

[8] D. A. Drew, R. T. Lahey, Jr., Analytical modeling of multiphase flow, In: Particulate Two-phase Flow (1993) 509-566.

[9] M. Lopez De Bertodano, R. L. Jr., O. Jones, Development of a k- $\epsilon$ model for bubbly two-phase flow, J. Fluids Eng. 13 (1987) 327-343.

[10] M. Lopez De Bertodano, R. L. Jr., O. Jones, Phase distribution in bubbly two-phase flow in vertical ducts., Int. J. Multiphase Flow 20 (1994) 805818.

[11] T. C. Kuo, C. Pan, C. C. Chieng, Eulerian-Lagrangian computations on phase distribution of two-phase bubbly flows, Int. J. Numer. Meth. Fluids 24 (1997) 579-593.

[12] S. Guet, G. Ooms, R. Oliemans, Aiche j., Simplified two-fluid model for gas-lift efficiency predictions 51 (2005) 1885-1896.

[13] M. Ma, J. Lu, G. Tryggvason, Using statistical learning to close two-fluid multiphase flow equations for a simple bubbly system, Physics of Fluids (1994-present) 9 (2015) 092101.

[14] G. Huffman, C. Jones, R. Brodkey, Optimization of turbulence models by means of a logical search algorithm, Applied Scientific Research 27 (1) (1973) 321-334.

[15] C. Lee, J. Kim, D. Babcock, R. Goodman, Application of neural networks to turbulence control for drag reduction, Physics of Fluids 9 (6) (1997) 1740-1747. 
[16] M. Milano, P. Koumoutsakos, Neural network modeling for near wall turbulent flow, Journal of Computational Physics 182 (2002) 1-26.

[17] E. Rajabi, M. R. Kavianpour, Intelligent prediction of turbulent flow over backward-facing step using direct numerical simulation data, Engineering Applications of Computational Fluid Mechanics 6 (4) (2012) 490-503.

[18] T. A. Oliver, R. D. Moser, Bayesian uncertainty quantification applied to rans turbulence models, in: Journal of Physics: Conference Series, Vol. 318, IOP Publishing, 2011, p. 042032.

[19] S. H. Cheung, T. A. Oliver, E. E. Prudencio, S. Prudhomme, R. D. Moser, Bayesian uncertainty analysis with applications to turbulence modeling, Reliability Engineering \& System Safety 96 (9) (2011) 1137-1149.

[20] J. Ling, J. Templeton, Evaluation of machine learning algorithms for prediction of regions of high reynolds averaged navier stokes uncertainty, Physics of Fluids (1994-present) 27 (8) (2015) 085103.

[21] F. Sarghini, G. de Felice, S. Santini, Neural networks based subgrid scale modeling in large eddy simulations, Computers \& Fluids (2003) 97-108.

[22] A. Moreau, O. Teytaud, J.-P. Bertoglio, Optimal estimation for large-eddy simulation of turbulence and application to the analysis of subgrid models, Physics of Fluids 18 (10) (2006) 105101.

[23] I. Esau, G. Rieber, On application of artificial neural network methods in large-eddy simulations with unresolved urban surfaces, Modern Applied Science 4 (2010) 3-11.

[24] S. Yarlanki, B. Rajendran, H. Hamann, Estimation of turbulence closure coefficients for data centers using machine learning algorithms, in: Thermal and Thermomechanical Phenomena in Electronic Systems (ITherm), 2012 13th IEEE Intersociety Conference on, IEEE, 2012, pp. 38-42. 
[33] A. Tomiyama, H. Tamai, I. Zun, S. Hosokawa, Transverse migration of single bubbles in simple shear flows, Chem. Eng. Sci. 57 (2002) 1849-1858.

[34] A. Kariyasaki, Behavior of a single gas bubble in a liquid flow with a linear velocity profile, in: Proceedings of the 1987 ASME-JSME Thermal

[25] B. Tracey, K. Duraisamy, J. J. Alonso, A machine learning strategy to assist turbulence model development, in: AIAA Paper 2015-1287, 2015.

[26] K. Duraisamy, Z. J. Zhang, A. P. Singh, New approaches in turbulence and transition modeling using data-driven techniques, in: AIAA Paper 2015$1283,2015$.

[27] E. J. Parish, K. Duraisamy, A paradigm for data-driven predictive modeling using field inversion and machine learning, Journal of Computational Physics 305 (2016) 758-774.

[28] C. Lu, S. Sambasivan, A. Kapahi, H. Udaykumar, Multi-scale modeling of shock interaction with a cloud of particles using an artificial neural network

[29] O. Sen, S. Davis, G. Jacobs, H. Udaykumar, Evaluation of convergence behavior of metamodeling techniques for bridging scales in multi-scale multimaterial simulation, Journal of Computational Physics 294 (2015) 585-604.

[30] J. Lu, S. Biswas, G. Tryggvason, A DNS study of laminar bubbly flows in a vertical channel, Int. J. Multiphase Flow 32 (2006) 643-660.

[31] A. Rust, M. Manga, Orientation and deformation of bubbles in shear flows, Journal of Colloid and Interface Science 249 (2002) 476-480.

[32] R. Zenit, Y. H. Tsang, D. L. Koch, A. S. Sangani, Shear flow of a suspension of bubbles rising in an inclined channel, Journal of Fluid Mechanics 515 (2004) 261-292. Engineering Joint Conference, Vol. 5, 1987, pp. 261-267. 
[35] E. Ervin, G. Tryggvason, The rise of bubbles in a vertical shear flow, ASME J. Fluid Engineering 119 (1997) 443-449.

[36] K. Sankaranarayanan, X. Shan, I. G. Kevrekidis, S. Sundaresan, Analysis of drag and virtual mass forces in bubbly suspensions using an implicit formulation of the lattice Boltzmann method, J. Fluid Mech. 452 (2002) $61-96$.

[37] J. Palacios, G. Tryggvason, The transient motion of buoyant bubbles in a vertical couette flow, AMD Contemporary Mathematics Series 466 (2008) $135-146$.

[38] G. Tryggvason, R. Scardovelli, Zaleski, Direct Numerical Simulations of Gas-Liquid Multiphase Flow, Cambridge University Press, 2011.

[39] M. P. Perrone, L. N. Cooper, When networks disagree: Ensemble methods for hybrid neural networks, in: R. J. Mammone (Ed.), Neural Networks for Speech and Image Processing,, Chapman and Hall, 1993.

[40] B. D. Ripley, Pattern Recognition and Neural Networks, Cambridge University Press, 1996.

[41] K. Tumer, J. Ghosh, Analysis of decision boundaries in linearly combined neural classifiers, Pattern Recognition 29 (2) (1996) 341-348.

[42] L. Breiman, Bagging predictors, Machine Learning 24 (1996) 123-140.

[43] C. M. Bishop, Neural networks for pattern recognition, Claridon Press, Oxford, 1995.

[44] M. Kuhn, K. Johnson, Applied predictive modeling, Springer,, 2013. 